\title{
Myeloid dendritic cells are decreased in peripheral blood of Alzheimer's disease patients in association with disease progression and severity of depressive symptoms
}

\author{
Antonio Ciaramella ${ }^{1 *}$ (D), Francesca Salani ${ }^{1}$, Federica Bizzoni ${ }^{1}$, Maria Donata Orfei ${ }^{1}$, Carlo Caltagirone ${ }^{1,2}$, \\ Gianfranco Spalletta ${ }^{1,3,4}$ and Paola Bossù ${ }^{1}$
}

\begin{abstract}
Background: Dendritic cells (DCs) are major orchestrators of immune responses and inflammation. They are migratory cells, which may play a role in Alzheimer's disease (AD), as suggested by prior in vitro studies. With the intent to investigate the clinical relevance of DC modifications in vivo, the present study was aimed to evaluate the levels of blood DCs in AD patients, in relation to the progression of the disease, the severity of its symptoms, and the treatment with acetylcholinesterase inhibitors (AChEls), a class of drugs used to improve cognitive functioning in people with dementia.

Methods: The two main subpopulations of immature blood DCs, namely myeloid ( $\mathrm{mDCs}$ ) and plasmacytoid (pDCs) cells, were evaluated by flow cytometry analysis in 106 AD patients, in comparison with the same cells from 65 individuals with mild cognitive impairment $(\mathrm{MCl})$ and 73 healthy control subjects $(\mathrm{HC})$. The relationship between blood DC levels and symptom severity was also assessed in AD patients, and their blood DC frequency was considered both in the absence or presence of treatment with AChEls.

Results: A significant depletion in blood mDCs was observed in AD patients, as compared to $\mathrm{HC}$ and $\mathrm{MCl}$ subjects. At variance, pDC levels were comparable among the three groups of subjects. The mDC decrease was evident only after the emergence of AD clinical symptoms, as confirmed by the follow-up analysis of a subgroup of $\mathrm{MCl}$ subjects who exhibited a significant decline in $\mathrm{mDC}$ after their conversion to AD. Notably, the $\mathrm{mDC}$ decline was inversely correlated in $\mathrm{AD}$ patients with the frequency and severity of depressive symptoms. Eventually, the mDC depletion was not observable in patients treated with AChEls.
\end{abstract}

Conclusions: Our results provide the first evidence that blood $\mathrm{mDC}$ levels are dysregulated in AD. This phenomenon appears mainly linked to AD progression, associated with stronger severity of AD-related symptoms, and influenced by AChEl treatment. Taken all together, these data suggest that blood mDCs may serve as a cell source to test disease-induced and treatment-related changes and support the innovative notion that DCs play a role in AD, as ultimate evidence of the immune system participation in disease progression.

Keywords: Alzheimer's disease, Mild cognitive impairment, Plasmacytoid dendritic cells, Myeloid dendritic cells

\footnotetext{
* Correspondence: a.ciaramella@hsantalucia.it

'Department of Clinical and Behavioral Neurology, IRCCS Santa Lucia

Foundation, Experimental Neuro-psychobiology Lab, Via Ardeatina 306,

00179 Rome, Italy

Full list of author information is available at the end of the article
} 


\section{Background}

In association with amyloid plaques, reactive microglia have been broadly observed in AD brain. These cells are considered the resident mononuclear phagocytes, which act as the first line of brain defense, like macrophages in the periphery, and are associated with the release of many inflammatory factors in AD brains, such as complement factors, pro-inflammatory cytokines and chemokines [1]. Similarly, pro-inflammatory mediators (mainly cytokines and chemokines) are also increased outside the CNS of AD patients and in particular in their peripheral blood [2]. Peripheral immune changes seem to occur in AD patients also at cellular level, especially regarding the myelomonocytic cells. Even though blood monocyte percentage appears similar in AD and controls [3], in vitro-stimulated AD monocytes acquire a pro-inflammatory phenotype with secretion of higher levels of IL-6 [4]. Moreover, monocytes obtained from $\mathrm{AD}$ patients have a limited potential to differentiate into macrophages, which, in turn, show an impaired phagocytosis of amyloid $\beta(A \beta)[5]$.

Following brain damage, an innate immune response takes place, mainly consisting of resident microglia and peripherally derived monocytes, macrophages [6], and, possibly, dendritic cells (DCs) [7]. Thus, it is not surprising that emerging data point to blood-derived mononuclear phagocytes as potential players in $\mathrm{AD}$, as shown in mouse models [8-11]. DCs are the most potent professional antigen-presenting cells that play a central role in the initiation and modulation of innate and adaptive immune response [12], but their role in $\mathrm{AD}$ context is still largely unexplored. DCs may play an important role in brain diseases as they can reach CNS from periphery in both human diseases [13] and animal models [14] including AD [7]. Unfortunately, the characterization of DCs in human CNS is hard because of their relative paucity in the brain parenchyma, the in vivo analysis is difficult to perform, and the phenotypic characterization is complex, due to the absence of specific markers that are able to clearly distinguish DCs from microglia. Notably, before entering tissues and exerting their specific biological activity, immature DCs may circulate in the blood, so their analysis in the periphery would provide insights to better understand their involvement in brain diseases. More specifically, blood DCs represent less than $1 \%$ of circulating mononuclear cells (PBMCs) in the peripheral blood, where they act as "immunological sentinels". Two major subsets of human blood DCs, myeloid and plasmacytoid DCs (mDCs and pDCs, respectively), have been classified $[15,16]$. Overall, both subsets of circulating DCs function as sentinels, which rapidly mature in response to antigenic stimulation, migrate to tissues, secrete cytokines, and effectively stimulate $\mathrm{T}$ cells. At variance with the $\mathrm{pDC}$ subset, $\mathrm{mDCs}$ are specifically considered precursor other than immature cells, since they fail to mature in response to TNF- $\alpha$ and express high levels of early myeloid markers, including CD33 [16]. Though the functional role of blood DCs and their subsets still needs to be clarified, a modification of their steady state has been often reported in ageing $[17,18]$ and in several pathological conditions, including CNS diseases and neurodegeneration [19-21], though not yet in AD. The knowledge about a potential involvement of $\mathrm{DCs}$ in $\mathrm{AD}$ is limited and mainly derived from in vitro data. We previously described that monocyte-derived DCs from AD patients show a more pronounced proinflammatory profile than DCs obtained from healthy control $(\mathrm{HC})$ subjects [3]. This phenomenon could be associated to altered metabolism of $A \beta$, since monocyte-derived DCs obtained from $\mathrm{HC}$ and generated in vitro with $A \beta_{1-42}$ peptide show functional alteration and increased production of inflammatory molecules [22]. In addition, we reported that monocyte-derived DCs from AD patients have a reduced ability to produce brain-derived neurotrophic factor following stimulation with $A \beta_{1-42}$ peptide [23], providing evidence in support of the concept that myeloid DCs might participate in AD brain damage by promoting both inflammatory response and $A \beta$-dependent neurotoxic pathways.

Given the migratory nature of DCs and their potential recruitment to brain during neurodegeneration, the present study was addressed to confirm the possible participation of $\mathrm{DCs}$ in $\mathrm{AD}$ by evaluating the levels of the two DC subsets present in the blood of AD patients, in comparison with $\mathrm{HC}$ subjects. Then, in order to assess when blood DC changes occur during the progression of dementia, the blood DC analysis was performed also in a group of individuals with primary memory impairments, namely amnestic mild cognitive impairment (MCI), who are considered at increased risk to develop $\mathrm{AD}$ [24], as well as in a subgroup of MCI who converted to $\mathrm{AD}$. With the intent to identify possible relationships between levels of blood DCs and clinical characteristics, the frequency of DC subpopulations in the blood was correlated with symptom severity in AD patients. Eventually, the impact on blood DC levels of acetylcholinesterase inhibitors (AChEIs), the drugs used as first line of symptomatic treatment of $\mathrm{AD}$, was also taken into consideration.

\section{Methods}

\section{Subjects}

This study focused on 244 subjects consecutively recruited in the outpatient memory clinic of Santa Lucia Foundation in Rome, Italy. Among those, 106 patients were diagnosed with "probable AD", 65 subjects with amnestic MCI, and 73 persons were included as $\mathrm{HC}$. 
Medical and psychiatric history were obtained from each subject, and all patients underwent a series of standard clinical evaluations, including physical, neurological, and mental status examinations and brain magnetic resonance imaging. The study was approved by the Santa Lucia Foundation Ethical Committee, and, in accordance with the Helsinki Declaration, written informed consent was obtained from patients, patient representatives, or caregivers prior to enrollment.

Common exclusion criteria applied to all subjects were (i) major medical illness (e.g., cancer, obstructive pulmonary disease or asthma, hematologic disorders, active gastrointestinal, renal, hepatic, endocrine, cardiovascular diseases) and autoimmune inflammatory disorders (e.g., rheumatoid arthritis, type I diabetes, psoriasis, systemic lupus erythematosus, overt infections); (ii) comorbidity of primary psychiatric (i.e., schizophrenia, major depression onset before the $\mathrm{AD}$ onset) or neurological disorders (i.e., stroke, Parkinson's disease (PD), seizure disorder, or head injury with loss of consciousness within the past year); (iii) known or suspected history of alcoholism or drug abuse; and (iv) computed tomography or magnetic resonance imaging evidence of focal parenchymal abnormalities.

Inclusion criteria for $\mathrm{AD}$ patients were (i) diagnostic evidence of probable AD consistent with the NINCDSADRDA criteria [25]; (ii) mild to moderate severity of dementia, defined as mini-mental state examination (MMSE) score ranging from 26 to 10 [26]; (iii) vision and hearing sufficient for compliance with testing procedures; and (iv) laboratory values within normal limits or considered not clinically relevant by the investigator.

Specific inclusion criteria for MCI were (i) diagnostic evidence of amnestic MCI consistent with Petersen guidelines [27]; (ii) a MMSE score $\geq 23$. MCI patients were at the onset of their cognitive impairment and underwent their first clinical examination for the diagnosis of MCI. To better establish whether blood DC modifications are specific of $\mathrm{AD}$ and when they occur during disease progression, $\mathrm{MCI}$ patients were followed for two consecutive years at 6-month clinical follow-up visits aimed at verifying conversion to $\mathrm{AD}$ or to confirm the MCI condition. A subgroup of $11 \mathrm{MCI}$ patients (16.9\% of the MCI cohort) who progressed over a 2-year time period to $A D$ was eventually identified within the main group, indicated as MCI converters. They had blood drawn and DC analysis both at MCI baseline condition and at conversion.

The HC were neither related to one another nor to $\mathrm{AD}$ patients. Their inclusion criteria were (i) vision and hearing sufficient for compliance with testing procedures; (ii) laboratory values within the appropriate normal reference intervals; and (iii) neuropsychological domain scores above the cutoff scores, corrected for age and educational level, identifying normal cognitive level in the Italian population. Specific exclusion criteria for $\mathrm{HC}$ subjects were (i) dementia diagnosis, according with DSM-IV criteria or MCI according with Petersen criteria, and confirmed by the administration of the MDB and (ii) MMSE score < 26 according with standardized norms for the Italian population [28].

\section{Demographic, cognitive, and neuropsychiatric assessment} Information on age, education, and sex was collected at baseline and verified by the patient's informant. Global cognitive functioning was assessed with the MMSE [26], a commonly used neurocognitive screening test addressed to make the first dementia diagnosis and to assess its progression and severity. MMSE measures orientation, language, verbal memory, attention, visuospatial function, and mental control, with scores ranging from 30 (no impairment) to 0 (maximum impairment).

To evaluate the severity of participants' neuropsychiatric symptoms, we administered to each participant the neuropsychiatric inventory (NPI) [29]. If appropriate, a family caregiver completed the NPI for the participant. The NPI was used to assess the frequency and severity of neuropsychiatric symptoms in 12 domains: delusions, hallucinations, agitation, depression, anxiety, euphoria, apathy, disinhibition, irritability, aberrant motor behavior, nighttime behavior disturbances, appetite, and eating abnormalities. Frequency was rated from 1 (occasionally) to 4 (very frequently) and severity from 1 (mild) to 3 (severe). If the symptom was absent, a score equal to 0 was given. The multiplication of frequency and severity was used as symptom composite score, with a range from 0 to 12 .

To assess the patients' ability to perform the activities of daily living independently, basic activities of daily living (ADL) were assessed using the Katz scale [30]. Instrumental ADL was assessed with Lawton and Brody's I-ADL scale [31]. The ADL and the I-ADL scales range from severe functional impairment (higher scores) to fully functioning (lower scores). They were treated as continuous variables.

Among the $106 \mathrm{AD}$ patients included in the study, 66 $(62.3 \%)$ were treated at baseline with the AChEI drug rivastigmine whereas 40 (37.7\%) were free from any AChEI treatment. Considering that taking AChEI may be a confounding factor for circulating immune cell analyses because of possible anti-inflammatory activity of these drugs, we split the AD group in the two subgroups of untreated (AD w/o AChEI) and AChEItreated patients.

Furthermore, at the moment of the clinical assessment and blood sampling collection, the following fraction of group subjects were depressed and treated with antidepressants: $12.3 \%$ of $\mathrm{MCI}$ and $10.3 \%$ of AD patients. In 
particular, blood donors utilized the following antidepressants: paroxetine $(1 / 65)$, sertraline $(3 / 65)$, citalopram $(1 / 65)$, and escitalopram (3/65) in $\mathrm{MCI}$ and paroxetine $(1 / 106)$, sertraline $(3 / 106)$, citalopram $(1 / 106)$, and escitalopram (6/106) in AD.

Baseline characteristics of the $\mathrm{HC}, \mathrm{MCI}$, and $\mathrm{AD}$ subjects used in this study, including AD subgroups, were reported in Table 1 . MCI subgroups, converters and noconverters, were described in Table 2. All groups were age and gender balanced according to chi-square test analysis (data not shown).

\section{Blood samples and flow cytometry analysis}

Peripheral blood samples were obtained by venipuncture, collected into 10-ml tubes (EDTA Vacutainer, BD Biosciences, San Diego, CA, USA), processed, and stained within $4 \mathrm{~h}$ of collection. The blood draws were done in the morning to avoid the influence of circadian rhythm on levels of circulating immune cells. The frequency of peripheral blood DCs was assessed by flow cytometry analysis according to the manufacturer's instructions and as described elsewhere [21]. In brief, $200 \mu \mathrm{L}$ of whole blood were incubated for $20 \mathrm{~min}$ at room temperature with antilin-1 FITC (i.e., CD3, CD14, CD16, CD19, CD20, and CD56), anti-HLA-DR PerCP, anti-CD11c APC, antiCD123 PE, and their isotype-matched monoclonal antibodies (all from Becton Dickinson, San Jose, CA, USA). Stained blood samples were subjected to erythrocytes lysis with $2 \mathrm{~mL}$ of FACS lysing solution (Becton Dickinson), vortexed and incubated for $10 \mathrm{~min}$ at RT in the dark. Following washing, samples were stored at $+4{ }^{\circ} \mathrm{C}$ in the dark and analyzed within $1 \mathrm{~h}$. Fifty thousand events were acquired with a four-color FACScalibur flow cytometer (BD Biosciences) running CellQuest software (BD Biosciences). Blood DCs were defined as lin- $1^{-} / \mathrm{HLA}^{-\mathrm{DR}^{++}}$cells. Blood DC subsets were identified using CD11c (mDCs) or CD123 (pDCs) antibodies. Representative panels that describe the gating strategy are reported in Fig. 1.

\section{Statistical analysis}

Data are shown in dot plots and expressed as mean values \pm SE. All statistical analyses were performed using Prism 4 software (GraphPad Software Inc). Comparisons between groups were made using the non-parametric two-tailed Mann-Whitney test for unpaired data or a two-tailed Wilcoxon signed rank test for paired data, as appropriate. Correlation analyses were made using Spearman's rank test. Results were considered to be statistically significant when $p$ value was $<0.05$.

\section{Results}

$\mathrm{mDC}$ percentage is decreased in peripheral blood from $\mathrm{AD}$ patients as compared to $\mathrm{MCl}$ and $\mathrm{HC}$ subjects

We firstly investigated by flow cytometry the relative proportion of peripheral blood DC subsets, more specifically $\mathrm{mDCs}$ and $\mathrm{pDCs}$, in the three main groups of subjects, namely $\mathrm{HC}, \mathrm{MCI}$, and $\mathrm{AD}$. The clinical characteristics of the three groups of subjects are summarized in Table 1. As reported in Fig. 2a, the mean percentage of $\mathrm{mDCs}$ was significantly decreased in $\mathrm{AD}$ patients $(0.20 \pm 0.009)$, as compared to $\mathrm{HC}(0.24 \pm 0.012, p=$ $0.01)$ or MCI $(0.26 \pm 0.011, p<0.0001)$, while no statistically significant differences were observed between $\mathrm{HC}$ and MCI subjects. There were no differences in levels of pDCs among AD, MCI, and $\mathrm{HC}$ (Fig. 2b).

\section{Blood $\mathrm{mDC}$ reduction is observed after conversion from $\mathrm{MCl}$ to $\mathrm{AD}$}

With the aim to better investigate the blood DC decline during $\mathrm{AD}$ progression, we have taken into consideration a subgroup of $\mathrm{MCI}$ converters and compared their peripheral blood DC frequency between before and after conversion. The clinical characteristics of the $\mathrm{MCI}$ groups of subjects at baseline are summarized in Table 2 . No main differences have been observed between the $\mathrm{MCI}$ converter subgroup and the whole MCI group as for age, education level, and gender distribution. As reported in Fig. 3a, most of the donors (9/11) showed a

Table 1 Demographic and clinical characteristics of $H C, M C l$, and $A D$ subjects

\begin{tabular}{llllll}
\hline & $H C$ & $M C l$ & $A D$ & AD w/o AChEl & AD AChEI \\
\hline$n$ & 73 & 65 & 106 & 40 & 66 \\
Gender (female, \%) & 57.2 & 57.0 & 51.9 & 72.0 & 70.0 \\
Age (years) & $71.5 \pm 0.5$ & $71.2 \pm 0.7$ & $8.5 \pm 0.5^{* *}$ & $9.0 \pm 0.7^{*}$ & $71.9 \pm 0.8$ \\
Education (years) & $11.3 \pm 0.5$ & $9.0 \pm 0.5^{*}$ & $20.9 \pm 0.4^{* *}$ & $22.0 \pm 0.6^{* *}$ & $8.1 \pm 0.7^{* *}$ \\
MMSE & $28.6 \pm 0.1$ & $27.0 \pm 0.2^{* *}$ & $8.5 \pm 0.3^{* *}$ & $8.0 \pm 0.3^{* *}$ & $20.0 \pm 0.6^{* *}$ \\
ADL & $5.9 \pm 0.3$ & $6.1 \pm 0.05$ & $13.8 \pm 0.6^{* *}$ & $12.9 \pm 0.8^{* *}$ & $8.9 \pm 0.5^{* *}$ \\
IADL & $6.7 \pm 0.2$ & $7.2 \pm 0.2$ & $3.0 \pm 0.2^{* *}$ & $3.4 \pm 0.4^{* *}$ & $14.6 \pm 0.8^{* *}$ \\
NPI dep. (F $\times S)$ & $1.3 \pm 0.2$ & $2.1 \pm 0.2^{*}$ & & $2.7 \pm 0.3^{* *}$ \\
\hline
\end{tabular}

Data are expressed as mean \pm SEM; ${ }^{*} p<0.005$ and ${ }^{* *} p<0.0005$ vs HC

MMSE mini-mental status examination, $A D L$ activities of daily living, IADL instrumental activities of daily living, $N P I$ dep. $(F \times S)$ neuropsychiatric

inventory-depression (frequency $\times$ severity) 
Table 2 Demographic and clinical characteristics of $\mathrm{MCl}$ subjects at baseline

\begin{tabular}{llll}
\hline & $\mathrm{MCl}$ total group & $\mathrm{MCl}$ converters & $\mathrm{MCl}$ no-converters \\
\hline$n$ & 65 & 11 & 54 \\
$\begin{array}{l}\text { Gender } \\
\text { (female, \%) }\end{array}$ & 57.0 & 45.5 & 55.5 \\
Age (years) & $70.1 \pm 0.7$ & $72.4 \pm 2.3$ & $70.9 \pm 0.7$ \\
Education (years) & $8.8 \pm 0.5$ & $8.6 \pm 1.2$ & $9.1 \pm 0.6$ \\
MMSE & $27.1 \pm 0.2$ & $26.9 \pm 0.7$ & $26.9 \pm 0.2$ \\
ADL & $6.1 \pm 0.04$ & $6.6 \pm 0.4$ & $6.1 \pm 0.04$ \\
IADL & $6.9 \pm 0.2$ & $8.0 \pm 0.8$ & $7.1 \pm 0.2$ \\
NPI dep. (F $\times S)$ & $1.9 \pm 0.2$ & $0.7 \pm 0.3^{*}$ & $2.4 \pm 0.3$ \\
pDCs (\%) & $0.073 \pm 0.005$ & $0.077 \pm 0.02$ & $0.072 \pm 0.005$ \\
mDCs (\%) & $0.26 \pm 0.011$ & $0.25 \pm 0.02$ & $0.26 \pm 0.012$ \\
\hline
\end{tabular}

Data are expressed as mean $\pm \mathrm{SEM} ;{ }^{*} p<0.05$ vs $\mathrm{MCl}$ total group and $\mathrm{MCl}$ no converters

decrease in $\mathrm{mDC}$ levels following $\mathrm{AD}$ conversion. Overall, the mean percentage of $\mathrm{mDCs}$ was significantly decreased in MCI converter $(0.25 \pm 0.020)$ only after their conversion to $\mathrm{AD}(0.17 \pm 0.021, p=0.002)$. Furthermore, the levels of $\mathrm{mDCs}$ were similar comparing the total group of MCI, the subgroup of MCI which is not converted to $\mathrm{AD}$ and the subgroup of $\mathrm{MCI}$ that converts to AD analyzed before conversion (Table 2). No differences in $\mathrm{pDC}$ values were identified in MCI converters between before and after conversion (Fig. 3b).

\section{Relationship between $\mathrm{mDC}$ levels and depression in $\mathrm{AD}$ patients}

Correlation analysis was performed in order to assess possible associations between frequencies of blood DCs and functional assessment of AD patients, such as cognitive deterioration and behavioral and psychological symptoms. A significant inverse correlation was found between $\mathrm{mDC}$ levels and depression symptoms $(n=91$, $p=0.01$, rho $=-0.25$ ). As shown in Fig. 4, blood DC levels were lower in patients with higher NPI scores, as regards in particular the frequency $\times$ severity of depressive symptoms. Interestingly, this correlation was significant in the case of $\mathrm{mDC}$ subset (Fig. 4a), while not for pDCs (Fig. 4b). No significant correlations were identified in AD patients between blood DC subsets and the other 11 NPI subscales, ADL, IADL, or MMSE scores (data not shown). Finally, the treatment of AD patients with antidepressants did not appear to influence the above-described correlation. In fact, similar to the whole group of $\mathrm{AD}$ patients, a significant inverse correlation between mDC levels and NPI score of depression was observed in $\mathrm{AD}$ patients who did not undergo to any depression medication not treated with antidepressant drugs (data not shown). No associations between blood DC subsets and symptom severity were identified in
MCI patients, both total group and MCI subgroups (data not shown).

\section{Blood $\mathrm{mDC}$ reduction is abated in $\mathrm{AD}$ patients treated with AChEI}

Since AD patients are commonly treated with cholinesterase inhibitors, which exert symptomatic effects and some delaying of disease progression, we ought to evaluate a potential interference of AChEI treatment on blood DCs frequency. Thus, we split our AD cohort into two subgroups, including AChEI-untreated and AChEItreated patients and their characteristics are summarized in Table 1. As shown in Fig. 5a, the mDC mean percentage in untreated $\mathrm{AD}$ patients (AD w/o AChEI; $0.16 \pm$ $0.014)$ was significantly decreased as compared to $\mathrm{HC}$ $(0.24 \pm 0.012, p<0.0001)$, as well as AD patients receiving AChEI therapy (AD AChEI; $0.22 \pm 0.011$ ). At variance, the $\mathrm{mDC}$ levels in the AChEI-treated subgroup of $\mathrm{AD}$ were only slightly lower than those observed in $\mathrm{HC}$, without any significant difference between them, suggesting that the anti-cholinesterase therapy may mitigate the blood $\mathrm{mDC}$ reduction observed in $\mathrm{AD}$ patients. No differences among the two groups of AChEI-untreated and AChEI-treated patients and $\mathrm{HC}$ were observed regarding the levels of blood pDCs (Fig. 5b).

\section{Discussion}

In recent years, DCs have emerged as a promising research area to investigate CNS diseases' pathophysiology and although a number of studies showed that DCs play critical roles in CNS inflammation, particularly during stroke or multiple sclerosis (MS) [20, 32], less evidence points to their potential role in neurodegenerative diseases. In this context, our data support the hypothesis that blood DCs are altered in AD patients.

Our main finding is that AD patients have decreased levels of the myeloid subset of blood DCs, as compared to matched HC. DC reduction is probably a general phenomenon in several full-blown diseases, since it has been featured in autoimmune diseases [33-35], infections [36], cancer [37], and CNS disturbances [19-21]. The blood DC reduction can be due to alterations in DC viability, DC mobilization, or their impaired differentiation from progenitors. Hence, the fate of DCs missing from the bloodstream is not always the same. In some cases, blood DCs specifically migrate from the blood to the diseased tissue. This situation was described in stroke or MS, where DCs have been found in the brain $[20,38]$, or in coronary artery disease, where DCs have been identified in atherosclerotic plaques [39]. On the contrary, DCs may die following infection, as in malaria [40], HIV infection [41], or severe sepsis [42], where infected DCs have been shown to undergo apoptosis. This latter circumstance seems unlikely to happen in 


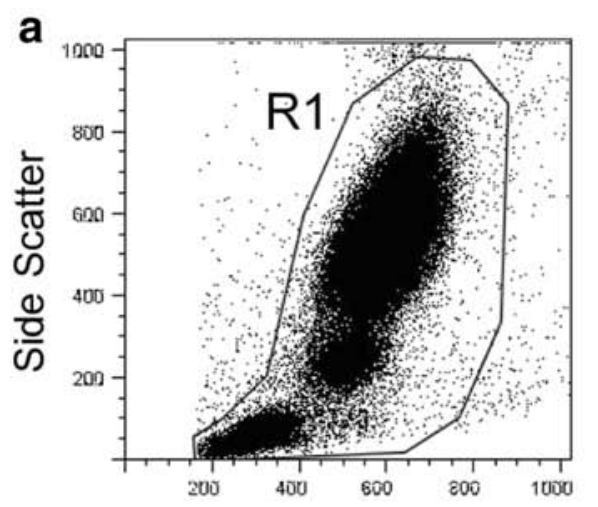

Forward Scatter
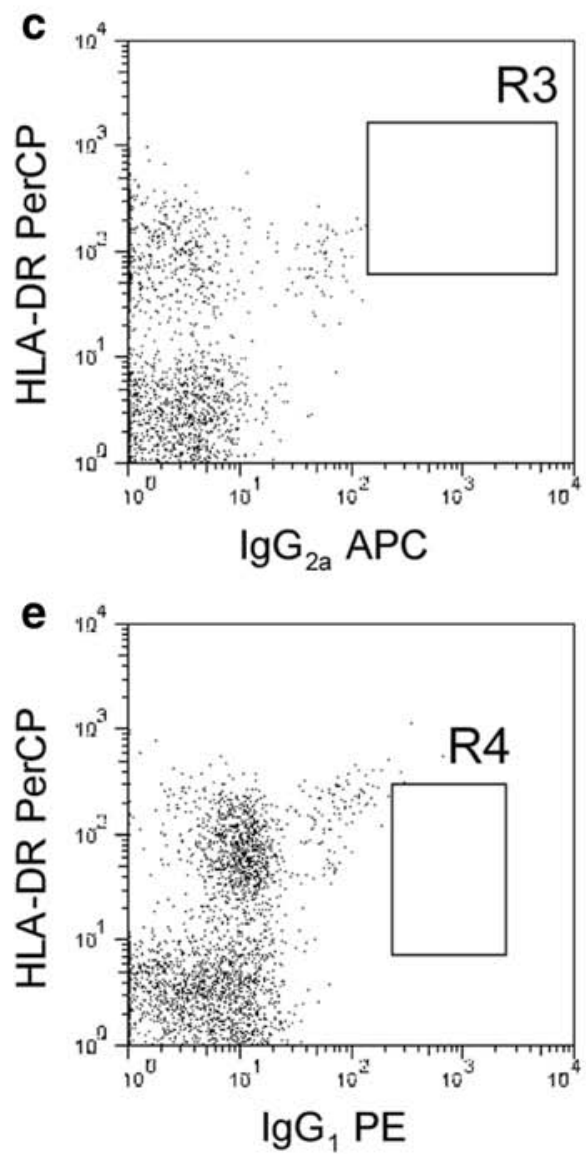

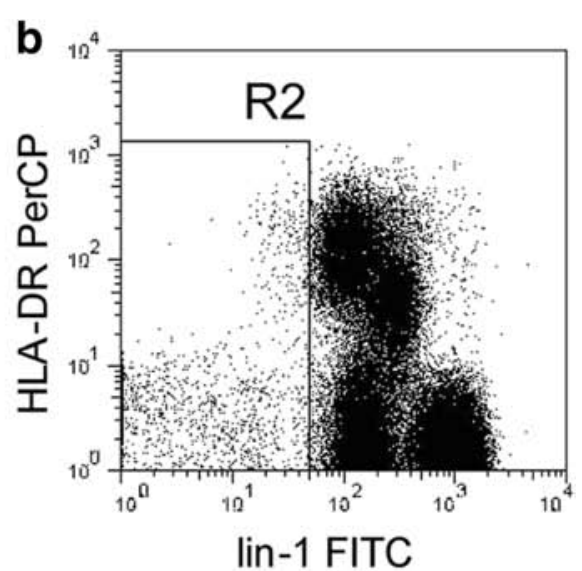

d
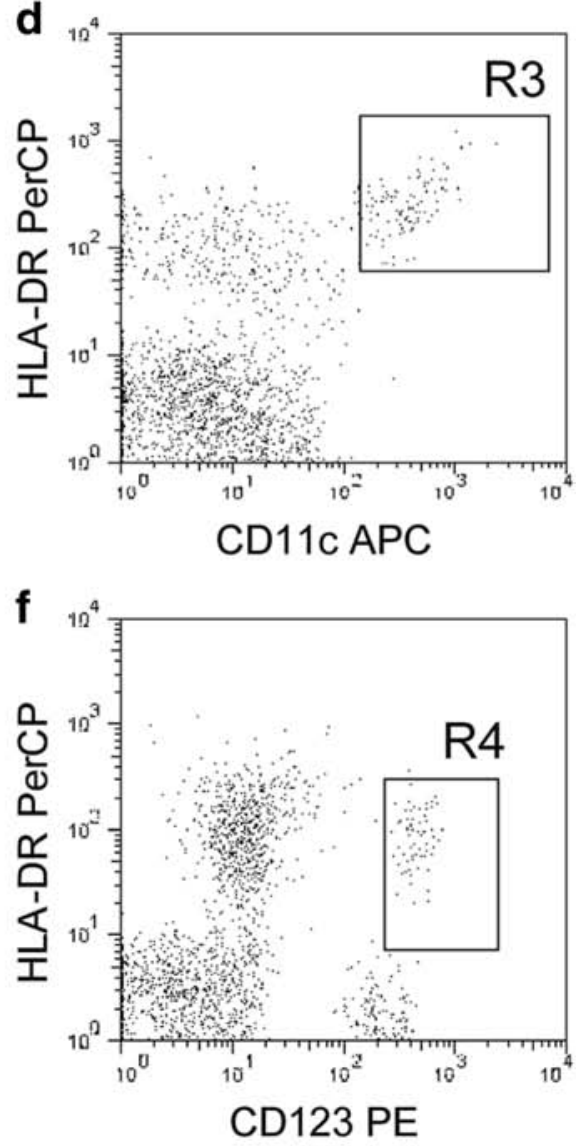

Fig. 1 Representative dot plots showing the gating strategy to identify mDCs and pDCs by flow cytometry. Whole peripheral leukocytes were selected from live cells based on forward scatter and side scatter characteristics (a, gate R1). Blood DCs were then selected within R1 positive cells as negative for lineage markers (lin-1) (b, gate R2). Finally, blood DCs were identified as mDC (d, HLA-DR $/$ CD $11 \mathrm{c}^{+} / \mathrm{CD} 123^{-}$, gate R3) or pDC (f, $\mathrm{HLA}_{-\mathrm{DR}}^{+} / \mathrm{CD} 11 \mathrm{C}^{-} / \mathrm{CD} 123^{+}$, gate R4) in comparison to respective isotype control monoclonal antibodies (c, e)

AD patients, where we did not observe an abnormal number of apoptotic DC in the peripheral blood (data not shown). Conversely, as previously demonstrated in stroke and MS, we speculate that in AD, a significant percentage of blood mDCs move from peripheral blood probably reaching the brain of patients, maybe at choroid plexus or meninges level, where DCs could sample cerebrospinal fluid content. To this regard, a part of $A \beta$ peptide efflux has been described to occur in AD exactly from these CNS elimination routes [43]. After DCs have reached these privileged positions, and sampled brain antigens, they may respond to neuroinflammation signals and react to neurodegeneration [44]. Importantly, the recent and accurate description of a CNS lymphatic 


\section{a}

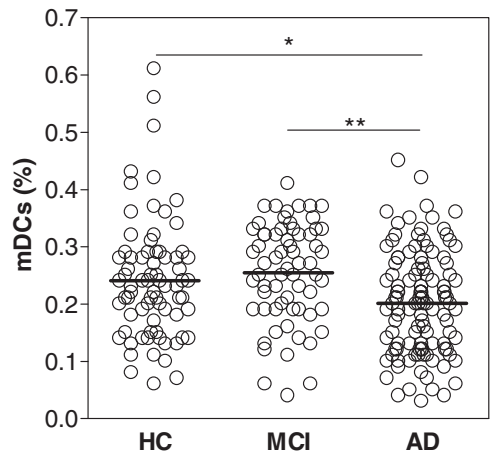

b

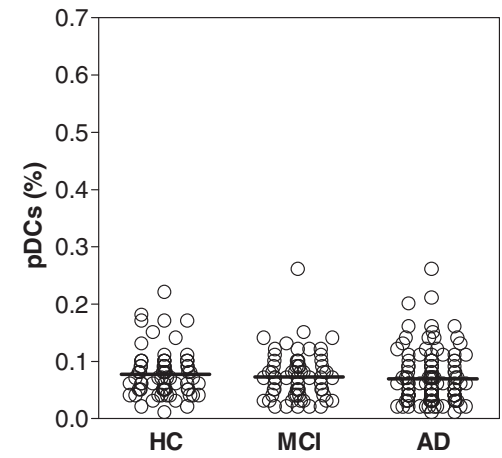

Fig. 2 Peripheral blood mDCs decline in AD patients. The distribution of percentage values of $\mathrm{mDCs}(\mathbf{a})$ and $\mathrm{pDCs}(\mathbf{b})$ present in the blood of HC $(n=73), \mathrm{MCl}(n=65)$, and $\mathrm{AD}(n=106)$ subjects is reported in the respective scatter dot plot, as indicated. The black bars indicate the mean. ${ }^{*} p=$ $0.01,{ }^{* *} p<0.0001$ (Mann-Whitney test)

system connected to deep cervical lymph nodes, which are able to drain cerebrospinal fluid and carry immune cells such as $\mathrm{CD}_{11 \mathrm{c}^{+}}[45]$, leads to more thorough investigation of the leukocyte presence in CNS immune responses in neurodegeneration and supports our hypothesis about blood-derived $\mathrm{DC}$ involvement in $\mathrm{AD}$ brain pathology.

Notably, we observed a specific reduction of myeloid DCs, while pDC subset appears mostly unchanged. This is in line with recent GWAS studies where the involvement of numerous immune-related genes as risk factors for $\mathrm{AD}$ was highlighted. Among these genes, there are CD33 and TREM2 notoriously associated with myeloid cell function. These studies strongly suggest that myeloid compartment of the immune system is a crucial component of susceptibility to AD [46]. Although it is still unclear to what extent peripheral myeloid cells engraft in the AD brain, it has been observed that CD33 expression levels are increased in CNS, but appeared decreased in peripheral mononuclear cells of $\mathrm{AD}$ patients
[47], suggesting that central and peripheral myeloid cell frequency may be linked processes during the disease. Furthermore, the specific implication of the $\mathrm{mDC}$ subset in $A D$ is consistent with a more pronounced pro-inflammatory feature of this subpopulation as compared to $\mathrm{pDCs}$ [48], also converging with our previous in vitro studies, which showed that monocyte-derived DCs acquire a pro-inflammatory profile in AD conditions $[3,22,23]$.

Differently from $A D$, we recently reported that in $\mathrm{PD}$ patients, there is a reduction of blood DCs both in $\mathrm{mDCs}$ and $\mathrm{pDCs}$ [21]. This last observation suggests that $\mathrm{mDC}$ decrease in the blood may be a common phenomenon in patients with neurodegenerative diseases, though DC subsets may be endowed with different functions in neuroinflammatory pathway linked to neurodegeneration evolution, depending on the type of specific neurological disturbance.

In the present study, we propose that blood $\mathrm{mDC}$ decrease appears specifically linked to $\mathrm{AD}$ progression, since
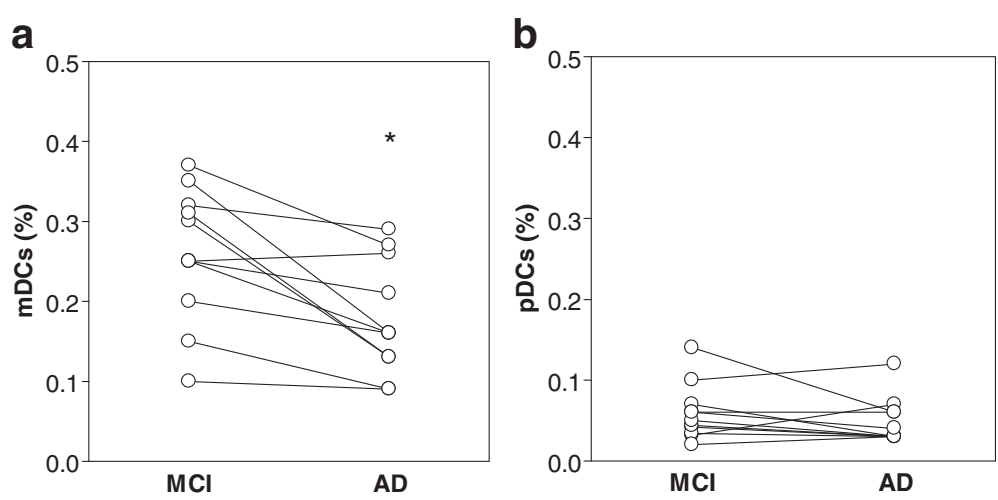

Fig. 3 Peripheral blood mDCs decline with AD conversion. "Before and after" graph showing levels of mDCs (a) and pDCs (b) in a subgroup of $11 \mathrm{MCl}$ converting to AD during a follow-up of 2 years. ${ }^{*} p=0.002$ (Wilcoxon matched paired test) 

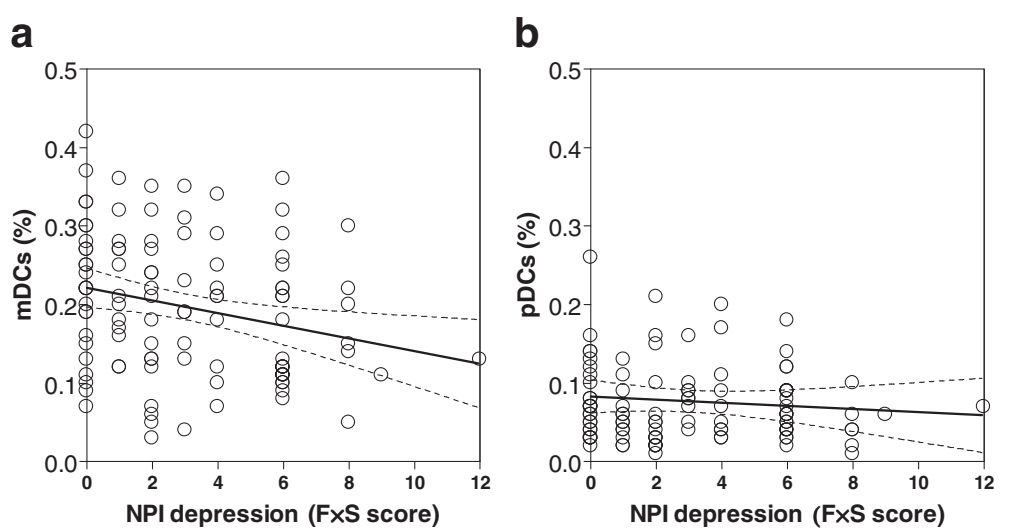

Fig. 4 Relationship between depression symptoms scores and levels of mDCs in AD patients. The correlation between depression symptoms scores (NPI) and percentage values of either mDCs (a) or pDCs $(\mathbf{b})$ present in the blood of AD patients $(n=106)$ is reported in the respective scatter dot plot, as indicated. The graphics show the tendency (black lines) and the $95 \%$ confidence interval (dotted lines)

the immune cell perturbation becomes evident in MCI subjects only after their conversion to AD while it is not evident at MCI level. Further, the blood $\mathrm{mDC}$ decrease is associated with severity and frequency of the depressive episodes and comes out mitigated in patients treated with the AChEI drugs used to slow down disease progression.

In fact, regarding the MCI subjects, namely individuals at increased risk to develop dementia, we observed that $\mathrm{mDC}$ levels were not different from those observed in control donors. This was true not only for the whole group of MCI but also for MCI converters, the retrospectively identified subgroup of $\mathrm{MCI}$ patients who have subsequently converted to $\mathrm{AD}$, hence in the earliest phase of AD. This indicates that blood $\mathrm{mDC}$ depletion specifically occurs only after the onset of $\mathrm{AD}$ symptoms suggesting that $\mathrm{DCs}$ may be involved in $\mathrm{AD}$ progression, but not in the earlier phases of dementia.

We also found low $\mathrm{mDC}$ levels associated with increased depressive severity suggesting that depletion of $\mathrm{mDC}$ subset in $\mathrm{AD}$ may be a functional feature of neuroinflammation-dependent neurodegenerative symptoms. The possibility that DC alteration may be linked to depressive symptoms is not very surprising, since depression and dementia share many common pathophysiological features with chronic inflammation, activation of the hypothalamic-pituitary-adrenal axis and deficit of neurotrophin signalling [49].

Finally, we observed that reduction of blood mDCs did not occur in AD patients treated with AChEI. These drugs, recommended as first-line treatment option for
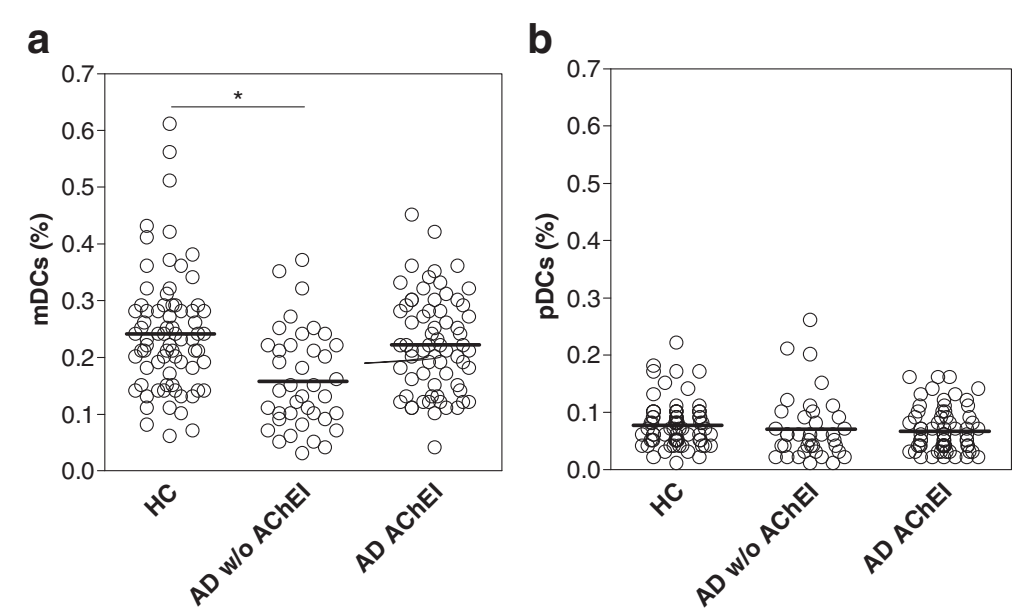

Fig. 5 Treatment with AChEl modulates the level of mDCs in AD patients. The distribution of percentage values of mDCs (a) and pDCs (b) present in the blood of $\mathrm{HC}(n=73)$, AD without AChEl treatment $(n=40)$, and AD treated with AChEl $(n=66)$ is reported in the respective scatter dot plot, as indicated. The black bars indicate the mean. ${ }^{*} p<0.0001$ 
patients with mild to moderate $\mathrm{AD}$, exert symptomatic effects and delay the natural course of the disease, supporting the hypothesis of an association between blood $\mathrm{mDC}$ perturbation and $\mathrm{AD}$ progression. Interestingly, AChEI treatment has been also reported to have beneficial effects on behavioural disturbances, including depression [50], further strengthening the suggested link between $\mathrm{mDC}$ frequency, $\mathrm{AD}$ progression, and depressive symptoms. In addition, AChEI, which are commonly used to compensate for acetylcholine depletion in $\mathrm{AD}$ brains, other than enhancing cholinergic transmission, may also exert anti-inflammatory activity on both neuroinflammation [51] and systemic inflammation [52]. Since a recent study showed that acetylcholine modulates functions of DCs cultured in vitro through an autocrine/ paracrine loop [53], further studies are highly recommended in order to identify the anti-inflammatory mechanisms of AChEI, DC-mediated.

\section{Limits of the study}

To our knowledge, this is the first study that analyzes the main subpopulations of circulating $\mathrm{DCs}$ in $\mathrm{AD}$ patients. Below are some points listed that should be investigated further. (i) We hypothesize that the main remark of this study, namely the reduction of $\mathrm{mDCs}$ from blood of $\mathrm{AD}$ patients, is associated with the recruitment of these cells in the brain. However, we did not investigate directly the fate of DCs, but we supposed their recruitment in the brain, taking in consideration previously reported studies [20,38]. Although clarifying the fate of myeloid DCs would allow to describe the phenotypic and functional features of these cells missing from the blood of AD patients, it is hindered by the difficulty to monitor the patients' brain for infiltrating myeloid cell subpopulations. (ii) We performed a cell staining strategy that allowed us to identify two different subpopulations of circulating DCs (lin-1/

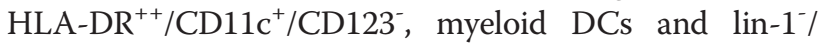
$\mathrm{HLA}^{-\mathrm{DR}}{ }^{++} / \mathrm{CD} 11 \mathrm{c}^{-} / \mathrm{CD} 123^{+}$, plasmacytoid DCs) but a more detailed DC characterization by BDCA's analysis [54] may prove useful to identify further distinctive DC subsets involved in AD. (iii) Finally, this study did not take into account the analysis of AD biomarkers and neuroimaging of brain inflammation, whose possible correlation with blood DC levels might add to our findings. The above limits are open questions which deserve to be clarified in future studies.

\section{Blood DCs as potential biomarkers}

In recent years, it has been shown that DCs may be a source of molecular marker for many diseases. For example, it has been shown that in lung tumors, mature DCs represent a specific marker for tumor-associated local lymph node-like structures [55] and, in some forms of leukemia, a specific subpopulation of BAD-LAMP ${ }^{+}$
pDCs identifies transformed pDCs [56]. In diabetes, it has been suggested that pDCs could predict Th1mediated type 1 disease [57] while, in systemic lupus erythematosus (SLE), there is strong genetic evidence that pDCs are critically involved in pathogenesis and autoantibody production [58]. The concept of immune processes as $\mathrm{AD}$ biomarker resource in neurodegenerative diseases is quite at the beginning. In particular, it has been shown that AD-derived monocytes exhibit an impaired differentiation into macrophages in vitro and also a limited phagocytic ability with a surface uptake of $A \beta$ undergoing apoptosis after $A \beta$ exposure [5]. Interestingly, a decreased abundance of monocytes was described in MCI compared with $\mathrm{AD}$ patients, suggesting a transient decline in the number of monocytes in the early stages of the disease [59]. More recently, lower levels of absolute percent of CD14 cells in early AD patients and an altered expression of HLA-DR, CD16, and CCR2 were found [60]. All studies reported above, including our findings, suggest that myeloid-derived cells, starting from monocytes to $\mathrm{DCs}$, are impaired in $\mathrm{AD}$, leading to ask what is the real contribution of these cells to the development of $\mathrm{AD}$ and whether they may be considered for further development of clinically relevant tools.

\section{Conclusions}

We identified blood mDCs as a cell population of high potential interest in $\mathrm{AD}$ progression and treatment, even though future longitudinal studies accompanied by evaluation of biomarkers, like those searching for $A \beta$ and tau proteins in cerebrospinal fluid, and specialized PET and MRI scans may be useful to better understand the meaning of $\mathrm{mDC}$ decline in $\mathrm{AD}$ clinical course.

In conclusion, this study provides the first evidence that blood DCs, specifically those of myeloid origin, are dysregulated in the blood of AD patients, fostering the concept that blood immune cells may play a key role in inflammatory-mediated progression of AD.

\section{Competing interests}

The authors declare that they have no competing interests.

\section{Authors' contributions}

$A C$ and PB conceived and designed the experiments. AC, FS, and FB performed the experiments. $A C$ and $P B$ analyzed the data. CC, GS, and MDO directed patient selection and clinical evaluations. $A C$ and PB wrote the paper. All authors made substantial contribution to analysis and discussion of the results. All authors contributed to the critical review of the manuscript. All authors read and approved the final version of the manuscript.

\section{Acknowledgements}

This study received financial support from Italian Ministry of Health, RC-13-14-15 (PB). This study was partially supported by the Italian Ministry of University, PRIN-2010PWNJXK (PB).

\section{Author details}

${ }^{1}$ Department of Clinical and Behavioral Neurology, IRCCS Santa Lucia Foundation, Experimental Neuro-psychobiology Lab, Via Ardeatina 306, 00179 Rome, Italy. ${ }^{2}$ Department of Neuroscience, University of Rome Tor 
Vergata, Via Montpellier 1, 00133 Rome, Italy. ${ }^{3}$ Neuropsychology Unit, University of Rome Tor Vergata, Via Montpellier 1, 00133 Rome, Italy. ${ }^{4}$ Menninger Department of Psychiatry and Behavioral Sciences, Baylor College of Medicine, Houston, TX, USA.

\section{Received: 27 July 2015 Accepted: 16 January 2016}

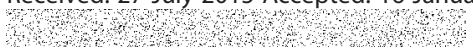

\section{References}

1. Akiyama H, Barger S, Barnum S, Bradt B, Bauer J, Cole GM, et al. Inflammation and Alzheimer's disease. Neurobiol Aging. 2000;21:383-421.

2. Swardfager W, Lanctôt K, Rothenburg L, Wong A, Cappell J, Herrmann N. A metaanalysis of cytokines in Alzheimer's disease. Biol Psychiatry. 2010;68:930-41.

3. Ciaramella A, Bizzoni F, Salani F, Vanni D, Spalletta G, Sanarico N, et al. Increased pro-inflammatory response by dendritic cells from patients with Alzheimer's disease. J Alzheimers Dis. 2010;19:559-72.

4. Shalit F, Sredni B, Stern L, Kott E, Huberman M. Elevated interleukin-6 secretion levels by mononuclear cells of Alzheimer's patients. Neurosci Lett. 1994;174:130-2.

5. Fiala M, Lin J, Ringman J, Kermani-Arab V, Tsao G, Patel A, et al. Ineffective phagocytosis of amyloid-beta by macrophages of Alzheimer's disease patients. J Alzheimers Dis. 2005:7:221-32. discussion 255-262.

6. Prinz M, Priller J. Microglia and brain macrophages in the molecular age: from origin to neuropsychiatric disease. Nat Rev Neurosci. 2014;15:300-12.

7. Butovsky $\mathrm{O}$, Kunis $\mathrm{G}$, Koronyo-Hamaoui M, Schwartz M. Selective ablation of bone marrow-derived dendritic cells increases amyloid plaques in a mouse Alzheimer's disease model. Eur J Neurosci. 2007;26(2):413-6.

8. Malm TM, Koistinaho M, Pärepalo M, Vatanen T, Ooka A, Karlsson S, et al. Bone-marrow-derived cells contribute to the recruitment of microglial cells in response to beta-amyloid deposition in APP/PS1 double transgenic Alzheimer mice. Neurobiol Dis. 2005;18:134-42.

9. Mildner A, Schlevogt B, Kierdorf K, Böttcher C, Erny D, Kummer MP, et al. Distinct and non-redundant roles of microglia and myeloid subsets in mouse models of Alzheimer's disease. J Neurosci. 2011;31:11159-71.

10. Michaud JP, Bellavance MA, Préfontaine P, Rivest S. Real-time in vivo imaging reveals the ability of monocytes to clear vascular amyloid beta. Cell Rep. 2013;5:646-53.

11. Hohsfield LA, Humpel C. Migration of blood cells to -amyloid plaques in Alzheimer's disease. Exp Gerontol. 2015;65:8-15.

12. Banchereau J, Briere F, Caux C, Davoust J, Lebecque S, Liu YJ, et al. Immunobiology of dendritic cells. Annu Rev Immunol. 2000;18:767-811.

13. Pashenkov M, Teleshova N, Link H. Inflammation in the central nervous system: the role for dendritic cells. Brain Pathol. 2003;13:23-33.

14. D'Agostino PM, Gottfried-Blackmore A, Anandasabapathy N, Bulloch K. Brain dendritic cells: biology and pathology. Acta Neuropathol. 2012;124:599-614.

15. Shortman K, Liu YJ. Mouse and human dendritic cell subtypes. Nat Rev Immunol. 2002;2:151-61.

16. Ziegler-Heitbrock L, Ancuta P, Crowe S, Dalod M, Grau V, Hart DN, et al. Nomenclature of monocytes and dendritic cells in blood. Blood. 2010; 116:e74-80

17. Della Bella S, Bierti L, Presicce P, Arienti R, Valenti M, Saresella M, et al. Peripheral blood dendritic cells and monocytes are differently regulated in the elderly. Clin Immunol. 2007;122:220-8.

18. Perez-Cabezas B, Naranjo-Gomez M, Fernandez MA, Grifols JR, Pujol-Borrell $\mathrm{R}$, Borras FE. Reduced numbers of plasmacytoid dendritic cells in aged blood donors. Exp Gerontol. 2007:42:1033-8.

19. Thewissen K, Nuyts AH, Deckx N, Van Wijmeersch B, Nagels G, D'hooghe M, et al. Circulating dendritic cells of multiple sclerosis patients are proinflammatory and their frequency is correlated with MS-associated genetic risk factors. Mult Scler. 2014;20:548-57.

20. Yilmaz A, Fuchs T, Dietel B, Altendorf R, Cicha I, Stumpf C, et al. Transient decrease in circulating dendritic cell precursors after acute stroke: potentia recruitment into the brain. Clin Sci (Lond). 2010;118:147-57.

21. Ciaramella A, Salani F, Bizzoni F, Pontieri FE, Stefani A, Pierantozzi M, et al. Blood dendritic cell frequency declines in idiopathic Parkinson's disease and is associated with motor symptom severity. PLoS One. 2013:8, e65352.

22. Ciaramella A, Sanarico N, Bizzoni F, Moro ML, Salani F, Scapigliati G, et al. Amyloid beta peptide promotes differentiation of pro-inflammatory human myeloid dendritic cells. Neurobiol Aging. 2009;30:210-21.
23. Ciaramella A, Salani F, Bizzoni F, Orfei MD, Langella R, Angelucci F, et al. The stimulation of dendritic cells by amyloid beta 1-42 reduces BDNF production in Alzheimer's disease patients. Brain Behav Immun. 2013:32:29-32.

24. Albert MS, DeKosky ST, Dickson D, Dubois B, Feldman HH, Fox NC, et al. The diagnosis of mild cognitive impairment due to Alzheimer's disease: recommendations from the National Institute on Aging-Alzheimer's Association workgroups on diagnostic guidelines for Alzheimer's disease. Alzheimers Dement. 2011;7:270-9.

25. Dubois B, Feldman HH, Jacova C, Dekosky ST, Barberger-Gateau P, Cummings J, et al. Research criteria for the diagnosis of Alzheimer's disease: revising the NINCDS-ADRDA criteria. Lancet Neurol. 2007;6:734-46.

26. Folstein MF, Folstein SE, McHugh PR. "Mini-mental state". A practical method for grading the cognitive state of patients for the clinician. J Psychiatr Res. 1975;12:189-98.

27. Petersen RC, Smith GE, Waring SC, Ivnik RJ, Kokmen E, Tangelos EG. Aging, memory, and mild cognitive impairment. Int Psychogeriatr. 1997; 9 Suppl 1:65-9.

28. Carlesimo GA, Caltagirone C, Gainotti G. The Mental Deterioration Battery: normative data, diagnostic reliability and qualitative analyses of cognitive impairment. The Group for the Standardization of the Mental Deterioration Battery. Eur Neurol. 1996;36:378-84.

29. Cummings JL. The neuropsychiatric inventory: assessing psychopathology in dementia patients. Neurology. 1997;48:510-6.

30. Katz S. Assessing self-maintenance: activities of daily living, mobility, and instrumental activities of daily living. J Am Geriatr Soc. 1983;31:721-7.

31. Lawton MP, Brody EM. Assessment of older people: self-maintaining and instrumental activities of daily living. Gerontologist. 1969;9:179-86.

32. Stasiolek M, Bayas A, Kruse N, Wieczarkowiecz A, Toyka KV, Gold R, et al. Impaired maturation and altered regulatory function of plasmacytoid dendritic cells in multiple sclerosis. Brain. 2006;129:1293-305.

33. Baumgart DC, Metzke D, Schmitz J, Scheffold A, Sturm A, Wiedenmann $B$, et al. Patients with active inflammatory bowel disease lack immature peripheral blood plasmacytoid and myeloid dendritic cells. Gut. 2005;54:228-36

34. Galgani M, Nugnes R, Bruzzese D, Perna F, De Rosa V, Procaccini C, et al. Meta-immunological profiling of children with type 1 diabetes identifies new biomarkers to monitor disease progression. Diabetes. 2013;62:2481-91.

35. Robak E, Smolewski P, Wozniacka A, Sysa-Jedrzejowska A, Robak T. Clinical significance of circulating dendritic cells in patients with systemic lupus erythematosus. Mediators Inflamm. 2004;13:171-80.

36. Cutler CW, Jotwani R, Pulendran B. Dendritic cells: immune saviors or Achilles' heel? Infect Immun. 2001;69:4703-8.

37. Ye F, Yu Y, Hu Y, Lu W, Xie X. Alterations of dendritic cell subsets in the peripheral circulation of patients with cervical carcinoma. J Exp Clin Cancer Res. 2010;29:78

38. Nuyts AH, Lee WP, Bashir-Dar R, Berneman ZN, Cools N. Dendritic cells in multiple sclerosis: key players in the immunopathogenesis, key players for new cellular immunotherapies? Mult Scler. 2013;19:995-1002.

39. Van Vre EA, Van Brussel I, Bosmans JM, Vrints CJ, Bult H. Dendritic cells in human atherosclerosis: from circulation to atherosclerotic plaques. Mediators Inflamm. 2011:2011:941396.

40. Pinzon-Charry A, Woodberry T, Kienzle V, McPhun V, Minigo G, Lampah DA, et al. Apoptosis and dysfunction of blood dendritic cells in patients with falciparum and vivax malaria. J Exp Med. 2013;210:1635-46.

41. Meyers JH, Justement JS, Hallahan CW, Blair ET, Sun YA, O'Shea MA, et al. Impact of HIV on cell survival and antiviral activity of plasmacytoid dendritic cells. PLoS One. 2007;2, e458.

42. Riccardi F, Della Porta MG, Rovati B, Casazza A, Radolovich D, De Amici M, et al. Flow cytometric analysis of peripheral blood dendritic cells in patients with severe sepsis. Cytometry B Clin Cytom. 2011;80:14-21.

43. Crossgrove JS, Li GJ, Zheng W. The choroid plexus removes betaamyloid from brain cerebrospinal fluid. Exp Biol Med (Maywood). 2005; 230:771-6.

44. Karman J, Ling C, Sandor M, Fabry Z. Dendritic cells in the initiation of immune responses against central nervous system-derived antigens. Immunol Lett. 2004;92:107-15.

45. Louveau A, Smirnov I, Keyes TJ, Eccles JD, Rouhani SJ, Peske JD, et al. Structural and functional features of central nervous system lymphatic vessels. Nature. 2015. 
46. Raj T, Rothamel K, Mostafavi S, Ye C, Lee MN, Replogle JM, et al. Polarization of the effects of autoimmune and neurodegenerative risk alleles in leukocytes. Science. 2014;344:519-23.

47. Hu N, Tan MS, Sun L, Jiang $T$, Wang $Y L$, Tan $L$, et al. Decreased expression of CD33 in peripheral mononuclear cells of Alzheimer's disease patients. Neurosci Lett. 2014;563:51-4.

48. Liu YJ. IPC: professional type 1 interferon-producing cells and plasmacytoid dendritic cell precursors. Annu Rev Immunol. 2005;23:275-306.

49. Caraci F, Copani A, Nicoletti F, Drago F. Depression and Alzheimer's disease: neurobiological links and common pharmacological targets. Eur J Pharmacol. 2010;626:64-71.

50. Spalletta G, Caltagirone C, Padovani A, Sorbi S, Attar M, Colombo D, et al. Cognitive and affective changes in mild to moderate Alzheimer's disease patients undergoing switch of cholinesterase inhibitors: a 6-month observational study. PLoS One. 2014;9, e89216.

51. Nizri E, Hamra-Amitay Y, Sicsic C, Lavon I, Brenner T. Anti-inflammatory properties of cholinergic up-regulation: a new role for acetylcholinesterase inhibitors. Neuropharmacology. 2006;50:540-7.

52. Borovikova LV, Ivanova $S$, Zhang $M$, Yang $H$, Botchkina GI, Watkins $L R$, et al. Vagus nerve stimulation attenuates the systemic inflammatory response to endotoxin. Nature. 2000;405:458-62.

53. Salamone G, Lombardi G, Gori S, Nahmod K, Jancic C, Amaral MM, et al. Cholinergic modulation of dendritic cell function. J Neuroimmunol. 2011;236:47-56.

54. Dzionek A, Fuchs A, Schmidt P, Cremer S, Zysk M, Miltenyi S, et al. BDCA-2, BDCA-3, and BDCA-4: three markers for distinct subsets of dendritic cells in human peripheral blood. J Immunol. 2000;165(11):6037-46.

55. Goc J, Germain C, Vo-Bourgais TK, Lupo A, Klein C, Knockaert S, et al. Dendritic cells in tumor-associated tertiary lymphoid structures signal a Th1 cytotoxic immune contexture and license the positive prognostic value of infiltrating CD8+ T cells. Cancer Res. 2014;74(3):705-15.

56. Defays A, David A, de Gassart A, De Angelis Rigotti F, Wenger T, Camossetto $V$, et al. BAD-LAMP is a novel biomarker of nonactivated human plasmacytoid dendritic cells. Blood. 2011;118(3):609-17.

57. Xia CQ, Peng R, Chernatynskaya AV, Yuan L, Carter C, Valentine J, et al. Increased IFN-a-producing plasmacytoid dendritic cells (pDCs) in human Th1-mediated type 1 diabetes: pDCs augment Th1 responses through IFN-a production. J Immunol. 2014;193(3):1024-34.

58. Sisirak V, Ganguly D, Lewis KL, Couillault C, Tanaka L, Bolland S, et al. Genetic evidence for the role of plasmacytoid dendritic cells in systemic lupus erythematosus. J Exp Med. 2014;211(10):1969-76.

59. Lunnon K, Ibrahim Z, Proitsi P, Lourdusamy A, Newhouse S, Sattlecker M, et al. Mitochondrial dysfunction and immune activation are detectable in early Alzheimer's disease blood. J Alzheimers Dis. 2012;30(3):685-710.

60. Zhang R, Miller RG, Madison C, Jin X, Honrada R, Harris W, et al. Systemic immune system alterations in early stages of Alzheimer's disease. J Neuroimmunol. 2013;256(1-2):38-42.

\section{Submit your next manuscript to BioMed Central and we will help you at every step:}

- We accept pre-submission inquiries

- Our selector tool helps you to find the most relevant journal

- We provide round the clock customer support

- Convenient online submission

- Thorough peer review

- Inclusion in PubMed and all major indexing services

- Maximum visibility for your research

Submit your manuscript at www.biomedcentral.com/submit
Biomed Central 